\title{
Oral nitrate supplementation to enhance pulmonary rehabilitation in COPD: ON-EPIC a multicentre, double-blind, placebo-controlled, randomised parallel group study
}

\author{
Matthew J Pavitt (1), ${ }^{1}$ Rebecca Jayne Tanner, ${ }^{1}$ Adam Lewis, ${ }^{1}$ Sara Buttery, ${ }_{1}^{1}$ \\ Bhavin Mehta, ${ }^{2}$ Helen Jefford, ${ }^{3}$ Katrina J Curtis, ${ }^{1}$ Winston A S Banya, ${ }^{1}$ Syed Husain, ${ }^{4}$ \\ Karnan Satkunam, ${ }^{3}$ Dinesh Shrikrishna, ${ }^{5}$ William Man, ${ }^{1,2}$ Michael I Polkey, ${ }^{1,2}$ \\ Nicholas S Hopkinson
}

- Additional material is published online only. To view please visit the journal online (http://dx.doi.org/10.1136/ thoraxjn-2019-214278).

${ }^{1}$ National Heart and Lung Institute, Royal Brompton Campus, Imperial College London, London, UK

${ }^{2}$ Respiratory Medicine, Royal Brompton and Harefield NHS Foundation Trust, London, UK ${ }^{3}$ Greenwich Adult Community Health Service, Oxleas NHS Foundation Trust, Dartford, UK ${ }^{4}$ Respiratory Medicine, Maidstone and Tunbridge Wells NHS Trust, Maidstone, UK ${ }^{5}$ Musgrove Park Hospital, Taunton and Somerset NHS Foundation Trust, Taunton, UK

Correspondence to Dr Nicholas S Hopkinson, National Heart and Lung Institute, Royal Brompton Campus, Imperial College London, London SW3 6HP, UK n.hopkinson@ic.ac.uk

Received 31 October 2019 Revised 23 March 2020 Accepted 15 April 2020 Published Online First 6 May 2020

Check for updates

(C) Author(s) (or their employer(s)) 2020. No commercial re-use. See rights and permissions. Published by BMJ.

To cite: Pavitt MJ, Tanner RJ, Lewis A, et al. Thorax

2020:75:547-555.

\section{ABSTRACT}

Rationale Dietary nitrate supplementation has been proposed as a strategy to improve exercise performance, both in healthy individuals and in people with COPD. We aimed to assess whether it could enhance the effect of pulmonary rehabilitation (PR) in COPD.

Methods This double-blind, placebo-controlled, parallel group, randomised controlled study performed at four UK centres, enrolled adults with Global Initiative for Chronic Obstructive Lung Disease grade II-IV COPD and Medical Research Council dyspnoea score 3-5 or functional limitation to undertake a twice weekly 8-week PR programme. They were randomly assigned (1:1) to either $140 \mathrm{~mL}$ of nitrate-rich beetroot juice (BRJ) $(12.9 \mathrm{mmol}$ nitrate), or placebo nitrate-deplete BRJ, consumed 3 hours prior to undertaking each PR session. Allocation used computer-generated block randomisation.

Measurements The primary outcome was change in incremental shuttle walk test (ISWT) distance. Secondary outcomes included quality of life, physical activity level, endothelial function via flow-mediated dilatation, fat-free mass index and blood pressure parameters.

Results 165 participants were recruited, 78 randomised to nitrate-rich BRJ and 87 randomised to placebo. Exercise capacity increased more with active treatment $(n=57)$ than placebo $(n=65)$; median (IQR) change in ISWT distance $+60 \mathrm{~m}(10,85)$ vs $+30 \mathrm{~m}(0,70)$, estimated treatment effect $30 \mathrm{~m}$ ( $95 \% \mathrm{Cl} 10$ to 40$) ; p=0.027$. Active treatment also impacted on systolic blood pressure: treatment group $-5.0 \mathrm{~mm} \mathrm{Hg}(-5.0,-3.0)$ versus control $+6.0 \mathrm{~mm}$ $\mathrm{Hg}(-1.0,15.5)$, estimated treatment effect $-7 \mathrm{~mm} \mathrm{Hg}$ $(95 \% \mathrm{Cl} 7$ to -20$)(\mathrm{p}<0.0005)$. No significant serious adverse events or side effects were reported.

Conclusions Dietary nitrate supplementation appears to be a well-tolerated and effective strategy to augment the benefits of PR in COPD.

Trial registration number ISRCTN27860457.

\section{INTRODUCTION}

A cardinal feature of COPD is limited exercise capacity which impacts on individuals' ability to complete daily activities. Pulmonary rehabilitation (PR) is a multimodal therapy in which education, nutritional and psychological interventions are

\section{Key messages}

What is the key question?

- Can dietary nitrate supplementation enhance the exercise benefits of pulmonary rehabilitation in COPD?

What is the bottom line?

- In a multicentre, double-blind, placebocontrolled, parallel group randomised controlled study, dietary nitrate supplementation in combination with pulmonary rehabilitation increased exercise capacity in COPD.

Why read on?

- Pulmonary rehabilitation is recommended by all current international guidelines for the treatment of COPD. Resources to deliver programmes are limited and approaches to maximise individuals' response and so maximise value are needed.

combined with exercise training. The evidence base for PR is well established ${ }^{1}$ and $\mathrm{PR}$ is recommended by all current international guidelines for the treatment of COPD. However, resources to deliver programmes are limited ${ }^{2}$ and approaches to optimise individuals' response and so maximise value are needed.

Nitric oxide (NO) is a key regulator of skeletal muscle blood flow, contractility, glucose and calcium handling and mitochondrial function. ${ }^{3}$ A landmark study of nitrate supplementation by Larsen et $a l^{4}$ found that acute supplementation reduced the oxygen cost of submaximal exercise in healthy individuals, a finding confirmed by subsequent trials. ${ }^{5}$ In a prior study we showed that acute dietary nitrate supplementation reduced the oxygen cost of submaximal exercise assessed via cycle ergometry, but did not increase exercise capacity, in people with COPD. ${ }^{6}$ Research from other groups found that dietary nitrate supplementation increased exercise capacity after acute, $^{78}$ and after 14 days $^{9}$ of treatment, and also decreased blood pressure in COPD. ${ }^{78}$ Conversely, other studies have found no effect on exercise 
capacity $^{10-12}$ or oxygen kinetics. ${ }^{12}$ We hypothesised that dietary nitrate supplementation, taken prior to each exercise session during a PR programme, would enhance the gains in exercise capacity achieved by people with COPD.

\section{METHODS}

\section{Trial design and oversight}

The oral nitrate to enhance pulmonary rehabilitation in copd (ON-EPIC) trial was a multicentre, double-blind, placebocontrolled, randomised parallel group study, comparing dietary nitrate supplementation to a matched placebo.

The study was registered prospectively on a publicly accessible database (www.isrctn.com).

\section{Participants}

People with Global Initiative for Chronic Obstructive Lung Disease (GOLD) grade II-IV COPD ${ }^{13}$ were recruited from the PR services at the Royal Brompton and Harefield NHS Foundation Trust (NW London), Oxleas NHS Foundation Trust (Woolwich, SE London), Musgrove Park Hospital (Somerset) and Maidstone and Tunbridge Wells NHS Trust (Kent), between 26 January 2015 and 13 November 2017, with last person last visit completed on 8 January 2018. Eligibility for PR was determined, in line with the National Institute for Health and Care Excellence guidelines, as a Medical Research Council (MRC) dyspnoea score of $\geq 3$ or other functional limitation defined as patient-reported decrements in basic physical functions.

Exclusion criteria for the study included clinical instability (ie, within 1 month of an acute exacerbation of COPD), significant comorbidity limiting exercise tolerance, significant renal impairment (estimated glomerular filtration rate $<50 \mathrm{~mL}$ / $\mathrm{min}$ ), hypotension (systolic blood pressure $<100 \mathrm{~mm} \mathrm{Hg}$ ), use of nitrate-based medicine or phosphodiesterase $\mathrm{V}$ inhibitors or other reason to benefit from nitrate supplementation (ischaemic heart disease and peripheral arterial disease).

\section{Interventions}

The active intervention was a commercially available concentrated beetroot juice (BRJ) product which is cut with organic lemon juice $(2 \%)$ and contained $0.8 \mathrm{~g}$ or $12.9 \mathrm{mmol}$ of nitrate $(140 \mathrm{~mL}$ of Beet-It SPORT shot, James White Drinks, Ipswich, UK). The placebo was $140 \mathrm{~mL}$ of the same beverage which had been run through an ion exchange column by the manufacturers to remove the nitrate. ${ }^{14}$ The placebo drink was identical in taste and appearance, with both causing beeturia.

Study participants were instructed to consume the intervention 3 hours prior to attending the exercise component of every PR session, because plasma nitrite $\left(\mathrm{NO}_{2}{ }^{-}\right)$reaches peak concentrations at 3 hours after ingestion. ${ }^{15}$ Throughout the trial period participants were asked to avoid the use of antibacterial mouthwash and chewing gum. These have been shown to reduce the oral facultative bacteria whose nitrate reductase activity is essential for metabolism of an oral nitrate load. ${ }^{16}$ Participants were also asked to avoid consuming food naturally high in nitrate in the period of 48 hours prior to the baseline and end of study visits only (see online supplementary appendix 1). This was to create as standardised a condition as possible, reducing differing levels of dietary nitrate consumption as a source of variation around the time that measurements were made. Prior to the end of study visit, participants were asked to match their food and caffeine ${ }^{17}$ consumption with baseline. Other than these two specific periods they were asked to maintain their ordinary diet throughout the study. Compliance was assessed via empty bottle collection (see online supplementary appendix 2).

\section{Pulmonary rehabilitation}

PR programmes were conducted according to the American Thoracic Society (ATS)/European Respiratory Society (ERS) PR statement. ${ }^{18}$ The exercise component involved 8 weeks of twice weekly supervised exercise as well as a programme of homebased exercises and a mixture of aerobic and strength training. The initial level of exercise was based on performance during a baseline incremental shuttle walk test (ISWT), with the intensity of exercise increased according to the participant's symptom tolerance as they progressed through the programme. Completion of PR was defined as having attended at least 12 of the possible 16 sessions. $^{1}$

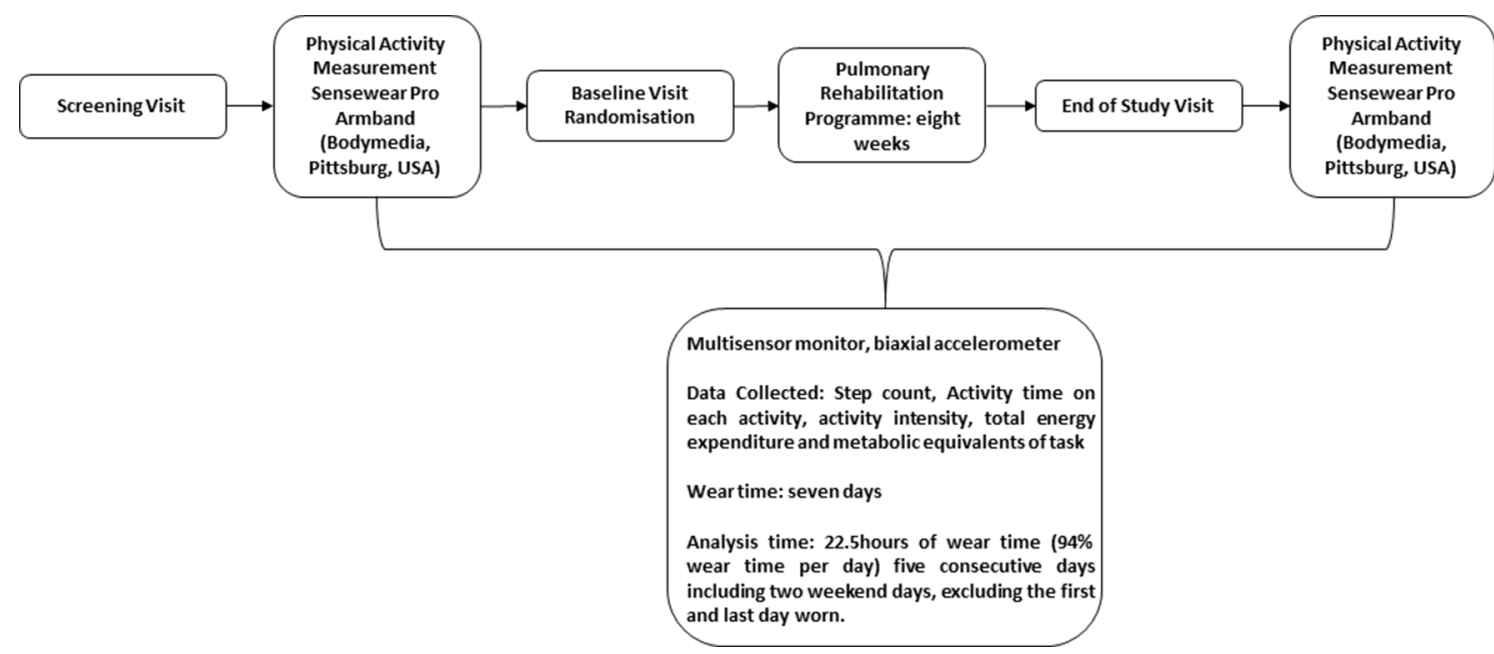

Figure 1 Physical activity measurement schematic. Individuals were requested to wear a SenseWear Pro Armband (BodyMedia, Pittsburgh, USA) following the screening visit and end of study visit. Armbands were worn for a period of 7 days. Data collected included step count, activity time on each activity, activity intensity, total energy expenditure and metabolic equivalents of task. Analysis was undertaken for 5 consecutive days with a wear time of 22.5 hours ( $94 \%$ wear time per day) including two weekend days and excluding the first and last days worn. 


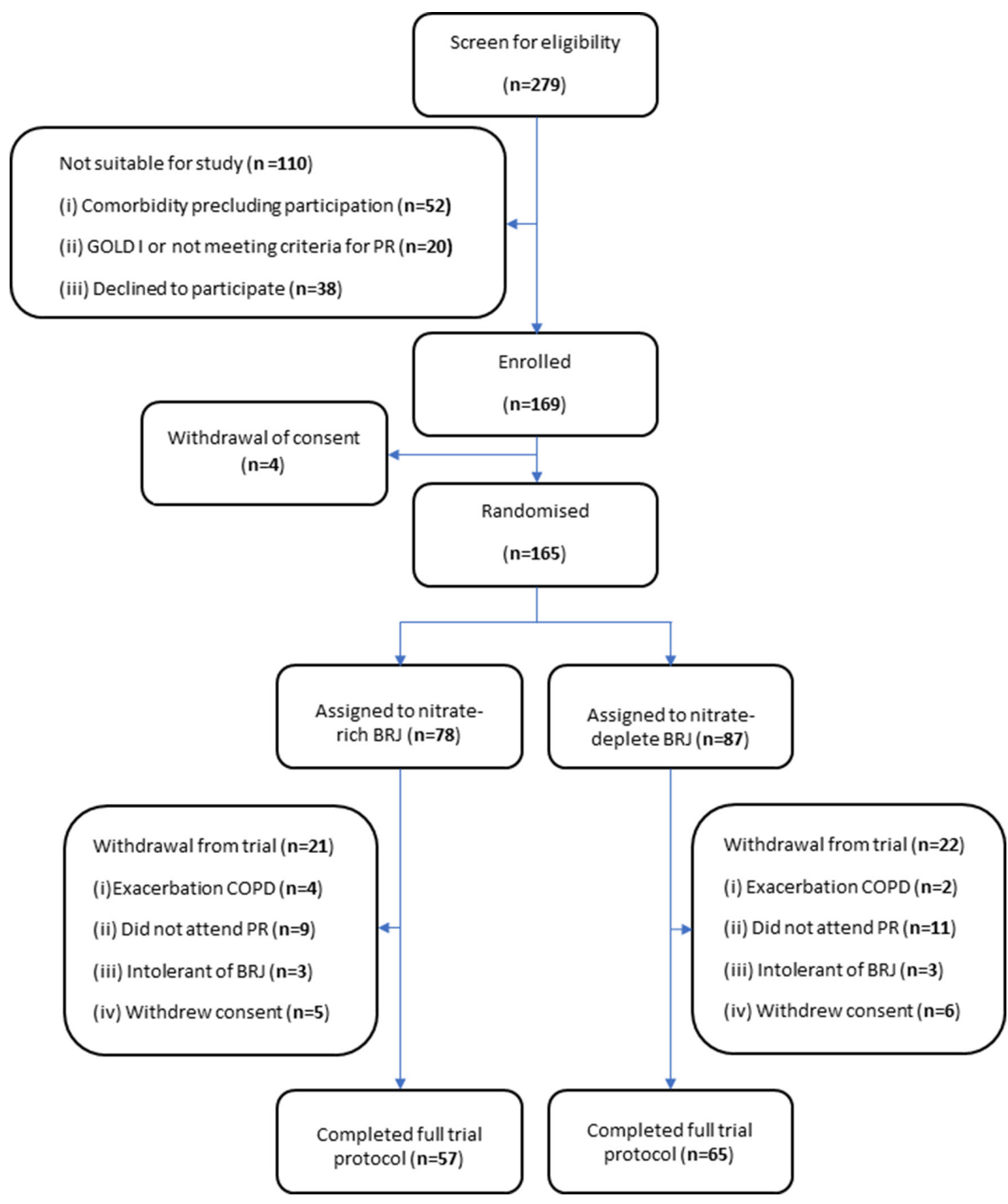

Figure 2 Consolidated Standards of Reporting Trials (CONSORT) recruitment diagram for enrolment and study completion. BRJ, beetroot juice; GOLD, Global Initiative for Chronic Obstructive Lung Disease; PR, pulmonary rehabilitation.

\section{Randomisation and blinding}

Individuals were randomised (1:1) using computer-generated block randomisation lists at each site, block size 10 , produced by an independent statistician with consecutive numbers linked to either intervention or placebo. Randomisation was performed following the screening visit after giving written consent and prior to baseline assessment. Both the outcome assessors and PR teams were blind to treatment allocation.

\section{Study conduct}

All study participants underwent a screening visit at their respective study centre to assess for eligibility and undertake spirometry.
Baseline assessments were undertaken within 7 days of screening, including vital signs, anthropometrics, quality of life, physical activity monitoring and ISWT. Assessments were repeated at an end of study visit a minimum of 7 days and maximum of 14 days after completing PR and the last dose of active or placebo BRJ. Outcome measures were standardised across centres.

\section{Primary outcome}

The primary outcome was change in ISWT from baseline, compared between study arms. A practice walk and repeat walk was performed with a rest of at least $30 \mathrm{~min}$ between them.

\begin{tabular}{llllll}
\hline \multicolumn{1}{l}{ Table 1} & \multicolumn{1}{l}{} \\
\hline & Trial completion and withdrawal rates by site & & & \\
& Combined & RBH & ONF & MPH & MTW \\
\hline Trial completers, $\mathrm{n}(\%)$ & $122(73.9)$ & $71(79.8)$ & $24(57.1)$ & $16(84.2)$ & $11(73.3)$ \\
Trial withdrawals, $\mathrm{n}(\%)$ & $43(26.1)$ & $18(20.2)$ & $18(42.9)$ & $3(15.8)$ & $4(26.7)$ \\
\hline
\end{tabular}

Data shown are number (\%).

MPH, Musgrove Park Hospital; MTW, Maidstone and Tunbridge Wells NHS Trust; ONF, Oxleas NHS Foundation Trust; RBH, Royal Brompton and Harefield NHS Foundation Trust. 
Table 2 Demographic and baseline clinical characteristics

\begin{tabular}{|c|c|c|c|}
\hline & $\begin{array}{l}\text { Placebo BRJ } \\
(n=65)\end{array}$ & $\begin{array}{l}\text { Nitrate-rich BRJ } \\
(\mathrm{n}=57)\end{array}$ & $P$ value \\
\hline Gender, n (\% female) & $26(41)$ & $27(42)$ & 1 \\
\hline Age (years) & $68(62,74)$ & $70(64,78)$ & 0.3 \\
\hline Smoking (pack-years) & $45(29,60)$ & $45(26,60)$ & 0.6 \\
\hline BMI $\left(\mathrm{kg} / \mathrm{m}^{2}\right)$ & $26(23,31)$ & $27(24,32)$ & 0.6 \\
\hline $\mathrm{FEV}_{1}(\mathrm{~L})$ & $1.1(0.8,1.4)$ & $1.2(1.0,1.6)$ & 0.4 \\
\hline $\mathrm{FEV}_{1}(\%$ predicted $)$ & $48(33,63)$ & $53(37,65)$ & 0.3 \\
\hline \multicolumn{4}{|l|}{ GOLD stage } \\
\hline $2, n(\%)$ & $27(42)$ & $31(54)$ & 0.4 \\
\hline $3, n(\%)$ & $24(37)$ & $20(35)$ & 1.0 \\
\hline $4, n(\%)$ & $14(21)$ & $6(11)$ & 1.0 \\
\hline FFM (kg) & $48.4(41.7,57.4)$ & $48.9(43.2,55.5)$ & 0.8 \\
\hline FFMI $\left(\mathrm{kg} / \mathrm{m}^{2}\right)$ & $17.1(15.5,19.8)$ & $17.5(15.9,19.2)$ & 0.8 \\
\hline Baseline ISWT (m) & $280(210,395)$ & $270(190,360)$ & 0.7 \\
\hline \multicolumn{4}{|l|}{ Inhalers } \\
\hline LABA, $n(\%)$ & $2(3)$ & $0(0)$ & 0.2 \\
\hline LAMA, n (\%) & $50(77)$ & $50(88)$ & 0.4 \\
\hline LABA-ICS, n (\%) & $39(60)$ & $38(67)$ & 0.1 \\
\hline LABA-LAMA, n (\%) & $7(11)$ & $2(4)$ & 0.1 \\
\hline MRC dyspnoea score & $3(3,3)$ & $3(3,3)$ & 0.7 \\
\hline CAT score & $19(13,23)$ & $20(13,24)$ & 0.9 \\
\hline HADS-A & $6(3,10)$ & $7(3,10)$ & 1.0 \\
\hline HADS-D & $5(3,9)$ & $5(3,8)$ & 1.0 \\
\hline Baseline systolic BP (mm Hg) & $130(120,145)$ & $135(124,152)$ & 0.2 \\
\hline Baseline diastolic BP (mm Hg) & $80(74,87)$ & $82(75,88)$ & 0.5 \\
\hline Baseline MAP (mm Hg) & $96(90,106)$ & $100(95,107)$ & 0.1 \\
\hline Baseline PAL & $3.0(2.6,3.8)$ & $3.0(2.4,4.3)$ & 0.9 \\
\hline Baseline step count (per day) & $3791(2271,6885)$ & $3507(1605,5314)$ & 0.3 \\
\hline Baseline time > 3 METs $(\mathrm{min})$ & $43(13,94)$ & $17(8,36)$ & 0.03 \\
\hline
\end{tabular}

Data shown are median (IQR) or number (\%). P values are for Mann-Whitney $\mathrm{U}$ test between groups.

BMI, body mass index; BP, blood pressure; BRJ, beetroot juice; CAT, COPD Assessment Test; FFM, fat-free mass; FFMI, fat-free mass index; GOLD, Global Initiative for Chronic Obstructive Lung Disease; HADS-A, Hospital Anxiety and Depression Scale-Anxiety; HADS-D, Hospital Anxiety and Depression Scale-Depression; ICS, inhaled corticosteroids; ISWT, incremental shuttle walk test; LABA, long-acting beta agonist; LAMA, long-acting muscarinic agonist; MAP, mean arterial pressure; METs, metabolic equivalents of task; MRC, Medical Research Council; PAL, physical activity level.

The furthest distance of the two walks at baseline and at study completion was used for analysis.

\section{Secondary outcomes}

Health-related quality of life was assessed using the COPD Assessment Test $(\mathrm{CAT})^{19}$ and psychological status using the Hospital Anxiety and Depression Scale (HADS). ${ }^{20}$ Breathlessness was assessed using the MRC dyspnoea score.

Blood pressure was measured using an automated monitor (Omron M6, Omron Healthcare Europe, Hoofddorp, Netherlands) at screening, baseline and end of study visits. After $10 \mathrm{~min}$ of seated rest, three measures were taken and the average values used. Height $(\mathrm{cm})$ was measured without shoes with a wallmounted measure, and weight $(\mathrm{kg})$ using standardised scales. Fat-free mass was determined using bioelectrical impedance (Bodystat 4000 device, Bodystat, Isle of Man, UK) and a diseasespecific regression equation. ${ }^{21}$

Physical activity measurement was performed using the SenseWear Pro Armband (BodyMedia, Pittsburgh, USA) which has been validated for use in people with COPD. ${ }^{22}{ }^{23}$ It is a multisensor monitor containing a biaxial accelerometer, worn continuously for a week except when bathing, over the bulk of the triceps on the upper left arm. Outputs include step counts, active time on each activity, activity intensity level, total energy expenditure and minutes of at least moderate activity (defined as any physical activity $>3$ metabolic equivalents of task (METs)). ${ }^{22}$ Data were collected following the screening visit (prior to the baseline assessment visit) and after the end of study visit. During each period participants were instructed to wear the activity monitor 24 hours/day for 7 days except while showering or bathing. The threshold for a valid day of activity was set at 22.5 hours (94\%) of wear time. Five consecutive valid days including two weekend days (Saturday and Sunday) were required to be included in the analysis (the first and last days of wearing not included $)^{24}$ (figure 1). Physical activity level (PAL) was calculated by dividing the estimated total daily energy expenditure by estimated wholenight sleeping energy expenditure. ${ }^{25}$ PAL definitions: active $\geq 1.70$; sedentary $1.40-1.69$; very inactive $<1.40$. $^{26}$ 


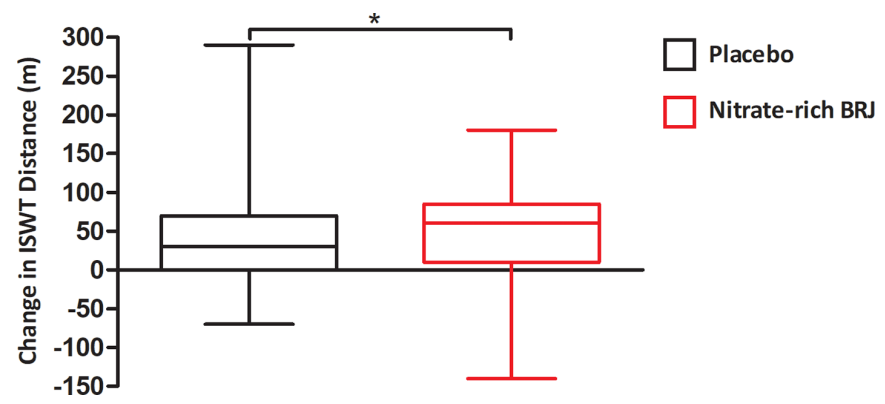

Figure 3 Primary outcome measure. Change ISWT distance $(\mathrm{m})$ in the placebo and nitrate-rich BRJ dosing conditions. Data presented as 25th-75th percentiles with the solid line representing the median value, and whiskers the minimum to maximum values. Mann-Whitney $U$ test was used to compare change in ISWT distance between treatment conditions; nitrate-rich BRJ $60 \mathrm{~m}(10,85)$ compared with placebo $30 \mathrm{~m}$ $(0,70) ;{ }^{*} p=0.027$. BRJ, beetroot juice; ISWT, incremental shuttle walk test.

Spirometry was measured in accordance with ATS/ERS statement on the standardisation of spirometry ${ }^{27}$ with a MicroLab spirometer (Vyaire Medical, Illinois, USA). Standardised lung function reference equations were based on the European Coal and Steel Community reference values. ${ }^{28}$

In addition, endothelial function was assessed using flowmediated dilatation (FMD) in a planned subset of 20 consecutive participants at the Royal Brompton Hospital only. FMD is a noninvasive, ultrasound-based evaluation of endothelial function in response to sheer stress. The diameter of the brachial artery was measured at baseline and sequentially after release of circulatory arrest of the upper arm. ${ }^{29}$ FMD is expressed as the per cent increase of peak arterial diameter following release of the cuff compared with the diameter at baseline. Analysis of FMD was undertaken blind to treatment allocation. Evaluation of plasma nitrate and nitrite levels had originally been planned, but due to logistical issues and limited resources it was not possible to undertake this within the study.

\section{Sample size}

This was a superiority trial, with nitrate-rich BRJ as the active intervention and nitrate-deplete BRJ as the control. To have an $80 \%$ statistical power, with a significance level of 0.05 , to detect an additional $20 \%$ improvement in walking distance, 61 subjects in each arm would need to complete the study. Allowing for a $15 \%$ withdrawal rate a sample size of 140 individuals was anticipated. The minimum clinically important difference (MCID) for the ISWT following PR defined as $47.5 \mathrm{~m}$ (95\% CI 38.6 to $56.5)$ at the time the study was planned ${ }^{30}$ though more recently a lower value of between 35 and $36.1 \mathrm{~m}$ has been established. ${ }^{31}$

\section{Statistical analysis}

Data analysis was conducted on an intention to treat basis (ie, per study arm allocation), in all participants who completed the full PR programme. Data are presented as mean (SD), or median (IQR) if not normally distributed. Differences in response between groups were assessed using unpaired t-test or MannWhitney U test as appropriate. Treatment effect was estimated using the Hodges-Lehmann estimates of shift parameters. Analysis was performed using SPSS V.24 for Windows and Stata V.16.1 (StataCorp. 2019 Stata Statistical Software: Release 16. College Station, TX: StataCorp). A p value $<0.05$ was considered to be statistically significant.

\section{RESULTS}

We screened 279 individuals for eligibility for the study (figure 2). Of these, 52 individuals had a comorbidity precluding entry, 20 had insufficiently severe COPD (GOLD grade I) or were insufficiently limited to meet the criteria for PR and 38 declined to participate in the study. After enrolment in the study four individuals withdrew consent prior to randomisation. In total, 165 individuals were randomised to receive nitrate-rich BRJ $(n=78)$ or placebo $(n=87)$. The imbalance was due to the use of separate randomisation lists at each site. After randomisation, 21 individuals withdrew from the nitrate-rich BRJ group and 22 individuals withdrew from the placebo group. Online supplementary appendix 3 shows baseline characteristics of trial completers and withdrawals. Table 1 shows trial completion and withdrawal rates by site. No participants had undertaken PR within 6 months of enrolling the study.

Table 2 shows the baseline characteristics for both subgroups, which were well matched, particularly for exercise capacitymedian baseline ISWT for nitrate-rich BRJ subgroup was $270 \mathrm{~m}$ $(190,360)$ versus placebo group $280 \mathrm{~m}(210,395)$. Six individuals (three in each arm) withdrew from the study due to intolerance of BRJ related to taste and gastrointestinal discomfort. All individuals were 100\% compliant with the dosing protocol as assessed by empty bottle count. No serious adverse effects were reported. Beeturia was almost universally reported. Among those completing the study, the mean (SD) number of PR sessions attended was 15 (2) sessions.

\section{Primary outcome}

Outcome measures were available for all 122 participants who completed the study unless otherwise specified. The median (IQR) improvement in ISWT distance following PR was statistically significant between the active treatment group compared with the placebo group, increasing by $60 \mathrm{~m}(10,85)$ compared with $30 \mathrm{~m}(0$, 70), estimated treatment effect $30 \mathrm{~m}$ (95\% CI 10 to 40$) ; \mathrm{p}=0.027$ (figure 3).

Table 3 Impact of pulmonary rehabilitation on symptoms and quality of life

\begin{tabular}{|c|c|c|c|c|c|}
\hline & Placebo BRJ $(n=64)$ & Nitrate-rich BRJ $(n=53)$ & Treatment effect & $95 \% \mathrm{Cl}$ & $P$ value \\
\hline$\triangle \mathrm{MRC}$ dyspnoea score & $0.0(-1.0,0.0)$ & $-1.0(-1.0,-0.0)$ & 0 & 0 to 0 & 0.90 \\
\hline$\triangle$ HADS-Anxiety & $-1.0(-3.0,0.8)$ & $-1.0(-2.0,0.3)$ & 0 & -1 to 1 & 0.58 \\
\hline$\triangle$ CAT score & $-2.0(-7.0,1.0)$ & $-2.5(-5.0,0.0)$ & 0 & -1 to 3 & 0.74 \\
\hline
\end{tabular}

Data shown are median (IQR). P values are for Mann-Whitney $\mathrm{U}$ test between study arms. Treatment effect was tested using Hodges-Lehmann estimates of shift parameters. BRJ, beetroot juice; CAT, COPD Assessment Test; HADS, Hospital Anxiety and Depression Scale; MRC, Medical Research Council. 


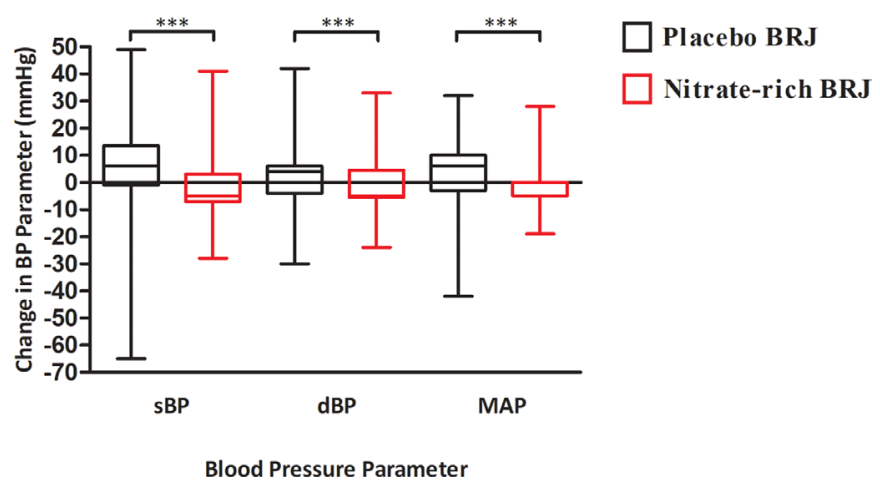

Figure 4 Change in blood pressure parameters $(\mathrm{mm} \mathrm{Hg})$ in the nitrate-rich BRJ and placebo dosing conditions. Data are presented as 25th-75th percentiles with the solid line representing the median value, and the whiskers the minimum to maximum values. Mann-Whitney $U$ test was used to compare blood pressure parameters. Blood pressure was measured at rest seated for $10 \mathrm{~min}$, with an average of three values reported. There was statistically significant reduction in systolic, diastolic and mean arterial blood pressure between dosing conditions. Change in systolic blood pressure in nitrate-rich BRJ group $-5.0 \mathrm{~mm} \mathrm{Hg}(-5.0$, $-3.0)$ versus placebo BRJ $+6.0 \mathrm{~mm} \mathrm{Hg}(-1.0,15.5), p=0.0005$. Change in diastolic blood pressure in nitrate-rich BRJ $-5.0 \mathrm{~mm} \mathrm{Hg}(-6.0,-2.0)$ versus placebo $B R J+6.0 \mathrm{~mm} \mathrm{Hg}(-4.5,7.5), p<0.0005$. Change in mean arterial pressure in nitrate-rich $B R J-5.0 \mathrm{~mm} \mathrm{Hg}(-5.0,-2.0)$ versus placebo BRJ $+6.0 \mathrm{~mm} \mathrm{Hg}(-3.0,11.0), \mathrm{p}<0.0005$. ${ }^{* *} \mathrm{P}<0.0005$. BP, blood pressure; BRJ, beetroot juice; $d B P$, diastolic blood pressure; MAP, mean arterial pressure; $s B P$, systolic blood pressure.

\section{Secondary outcomes}

No differences were observed between active and placebo treatments in MRC dyspnoea score, CAT score and HADS score following PR (table 3), although as expected both study groups experienced improvement compared with baseline. There was no statistically significant difference in the change in fat-free mass index between intervention groups; median (IQR) nitraterich $\mathrm{BRJ}+0.7 \mathrm{~kg} / \mathrm{m}^{2}(-1.7,2.0)$ versus placebo $-0.2 \mathrm{~kg} / \mathrm{m}^{2}$ $(-1.9,2.3)$, estimated treatment effect $0 \mathrm{~kg} / \mathrm{m}^{2}(95 \% \mathrm{CI}-0.7$ to 0.6$) ; \mathrm{p}=0.71$.

\section{Blood pressure responses}

The nitrate-rich BRJ group displayed a statistically significant improvement in systolic blood pressure at the end of PR compared with placebo; median (IQR) $-5.0 \mathrm{~mm} \mathrm{Hg}(-5.0$, $-3.0)$ vs $+6.0 \mathrm{~mm} \mathrm{Hg}(-1.0,15.5)$, estimated treatment effect $-7 \mathrm{~mm} \mathrm{Hg}(95 \% \mathrm{CI} 7$ to -20$) ; \mathrm{p}<0.0005$ (figure 4). Similar results were seen for diastolic blood pressure; $-5.0 \mathrm{~mm} \mathrm{Hg}$ $(-6.0,-2.0)$ vs $+6.0 \mathrm{~mm} \mathrm{Hg}(-4.5,7.5)$, estimated treatment effect $-7 \mathrm{~mm} \mathrm{Hg}(95 \%$ CI 0 to -14$) ; \mathrm{p}<0.0005$ and for mean arterial pressure; $-5.0 \mathrm{~mm} \mathrm{Hg}(-5.0,-2.0)$ vs $+6.0 \mathrm{~mm} \mathrm{Hg}$ $(-3.0,11.0)(p<0.0005)$. The absolute values for blood pressure parameters at baseline and end of study can be found in online supplementary appendix 4.

\section{Physical activity}

Because of issues with technical reliability and compliance relating to the use of SenseWear monitors only 65 paired sets of results were available (nitrate-rich BRJ $n=28$ and placebo $n=37$ ). Step count increased in the nitrate-rich BRJ group by median (IQR) 348 steps/day $(-94 ;+1629)$ and fell in the placebo group by 329 steps/day $(-915 ;+640)$, estimated treatment effect 784 steps/day (95\% CI 100 to 1471 ); $\mathrm{p}=0.02$ (figure 5). There was
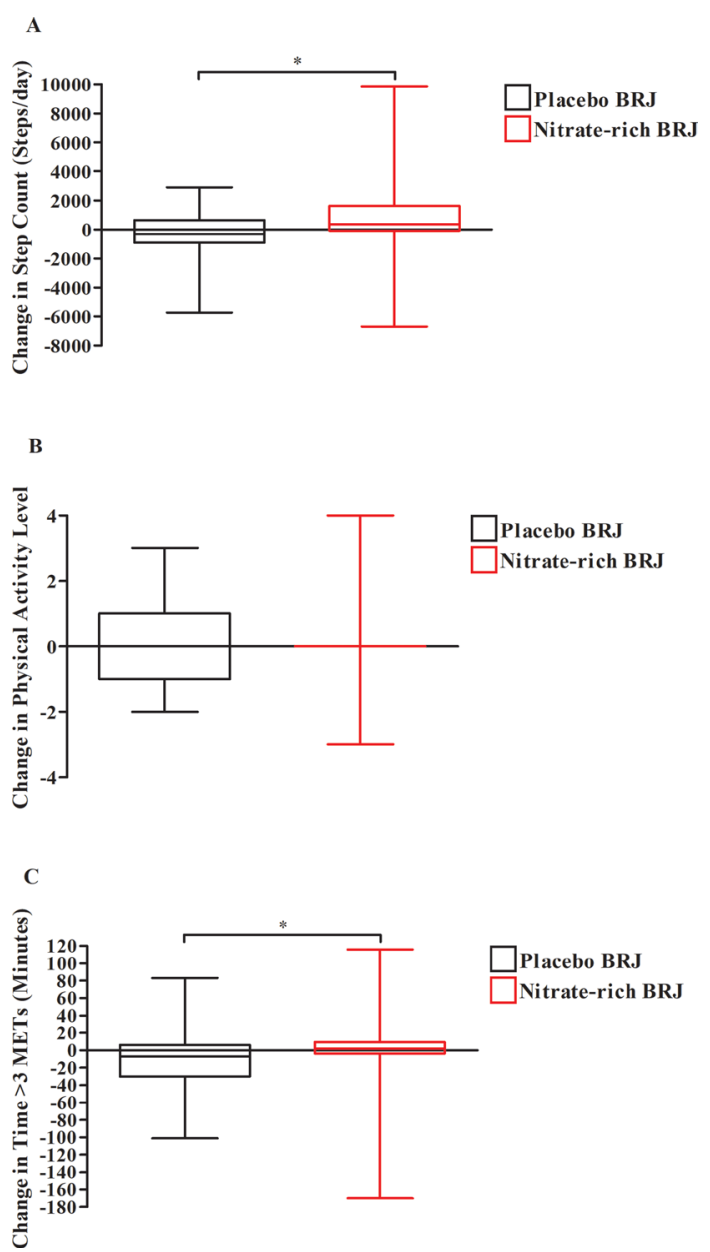

Figure 5 Change in measures of physical activity (step count, physical activity level and time spent in physical activity at greater than 3 METs). Data are presented as 25th and 75th percentiles with the solid line representing the median value, and the whiskers the minimum to maximum values. Mann-Whitney $U$ test was used to compare change in measures of physical activity. (A) Change in step count (steps/day) in the nitrate-rich BRJ and placebo BRJ dosing conditions. There was a statistically significant difference in step count; nitrate-rich BRJ 348 steps/day $(-94,1629)$ versus placebo BRJ -329 steps/day $(-915,640)$, ${ }^{*} p=0.02$. (B) Change in PAL in the nitrate-rich BRJ and placebo BRJ dosing conditions. There was no statistically significant difference in PAL; nitrate-rich BRJ $0.0(0.0,0.0)$ versus placebo BRJ $0.0(-1.0,1.0)$, $p=-0.73$. (C) Change in time spent in activity greater than 3 METs (min) in the nitrate-rich BRJ and placebo dosing conditions. There was a statistically significant difference in time spent in activity greater than 3 METs in the nitrate-rich BRJ $2.0 \mathrm{~min} /$ day $(-3.6,9.5)$ versus placebo BRJ $-7.0 \mathrm{~min} /$ day $(-30.0,6.0), p=0.02$. BRJ, beetroot juice; METs, metabolic equivalents of task; PAL, physical activity level.

no difference in change in PAL between groups; nitrate-rich BRJ $0.0(0.0 ; 0.0)$ versus placebo BRJ $0.0(-1.0 ; 1.0)$, estimated treatment effect $0.2(95 \% \mathrm{CI}-0.3$ to 0.7$) ; \mathrm{p}=0.73$, but there was a statistically significant difference in the change in time spent in activity >3 METs; nitrate-rich BRJ increasing by $2.0 \mathrm{minu} /$ day $(-3.6 ; 9.5)$ versus placebo BRJ falling by $7.0 \mathrm{~min} /$ day $(-30.0$; +6.0 ), estimated treatment effect $13 \mathrm{~min} /$ day (95\% CI 2 to 28 ); $\mathrm{p}=0.02$.

\section{Endothelial function}

In the planned substudy (nitrate-rich BRJ $n=10$, placebo BRJ $\mathrm{n}=10$ ) there was a statistically significant improvement in the 


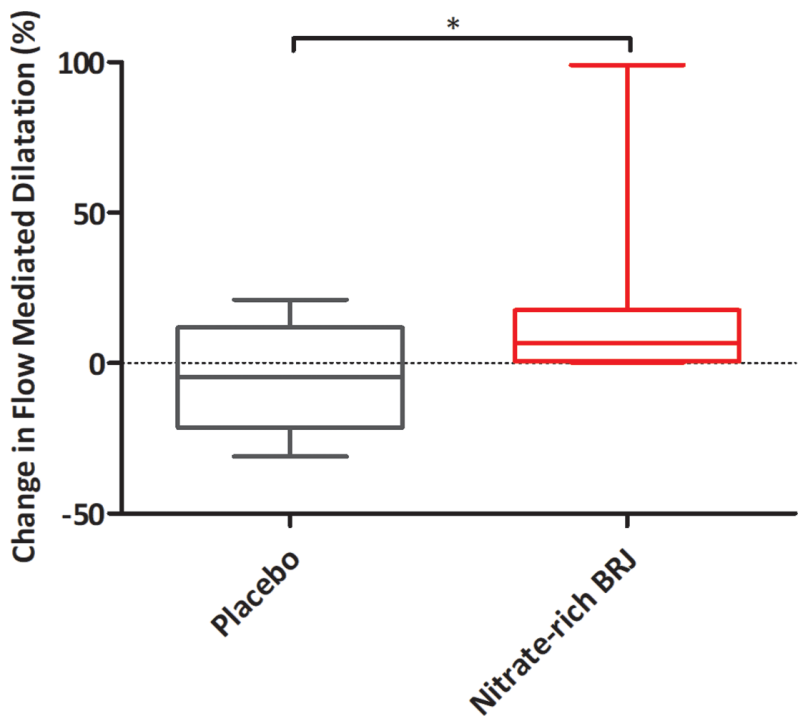

Figure 6 Change in flow-mediated dilatation percentage. Percentage change in flow-mediated dilatation (FMD \%). Data are presented as 25th and 75th percentiles with the solid line representing the median value, and the whiskers the minimum to maximum values. MannWhitney $\mathrm{U}$ test was used to compare the percentage change in FMD in the nitrate-rich BRJ and placebo dosing conditions. There was a statistically significant difference in FMD \% in the nitrate-rich BRJ group with an increase of $6.6 \%(0.6,17.6)$ versus a reduction in FMD \% in the placebo BRJ group $-4.7 \%(-21.5,11.8), p=0.046$. BRJ, beetroot juice.

median (IQR) per cent change in FMD in the nitrate-rich BRJ group $+6.6 \%(0.6,17.6)$ versus placebo BRJ $-4.7 \%(-21.5$, $11.8)$, estimated treatment effect $-20.3 \%$ (95\% CI -33.8 to $3.4) ; \mathrm{p}=0.046$ (figure 6).

\section{Adverse reactions}

There were no serious adverse events during this study. Equal numbers in each arm were unable to tolerate the BRJ due to gastrointestinal discomfort (nausea). Four (7\%) individuals in the intervention group and two $(3 \%)$ in the placebo group had a documented exacerbation of COPD (Mann-Whitney U test, $\mathrm{p}=0.550)$.

\section{DISCUSSION}

The main finding of this study was that, in people with COPD undertaking an 8-week course of PR, supplementation with nitrate-rich BRJ augmented the improvement in exercise capacity, measured as ISWT compared with an otherwise identical but nitrate-deplete placebo. Active treatment was also associated with reductions in blood pressure, greater daily step count and improved endothelial function compared with the control arm.

\section{Significance of findings}

PR is a high-value and cost-effective intervention, ${ }^{32}$ associated with significant health benefits including an improvement in exercise capacity, a reduction in hospital admissions, a reduced number of in-hospital bed-days and reduced mortality. ${ }^{33}$ The benefits of PR are thought to last approximately 12 months. ${ }^{34}$ However, access to PR is limited ${ }^{2}$ by resource availability and it is important to ensure the benefit of programmes is maximised. The present results suggest that dietary nitrate supplementation, a relatively cheap intervention with few side effects, can augment the improvement in exercise capacity produced by PR.
The estimated treatment effect of dietary nitrate supplementation on ISWT of $30 \mathrm{~m}(10,40)$ is close to but does not quite exceed the MCID for this measure, recently defined as between 35 and $36.1 \mathrm{~m},{ }^{31}$ however our findings are likely to be clinically relevant for several reasons. First, whether the benefit of an intervention is meaningful depends on the nature of the intervention. The level of acceptable response needed for a complex intervention like PR is higher than the response needed to justify the small additional cost and burden of consuming a beetroot shot twice a week. Second, the improvement in health status associated with PR is partly driven by psychological factors and social interaction through participation in PR distinct from direct exercise training effects, so a direct comparison of the effects is inappropriate as the nitrate supplementation would not be expected to effect the former category. Third, the finding that measures of physical activity improved with treatment compared with controls suggests an impact on daily activity as well as performance on exercise tests. The MCID for step count following PR is 600 steps/day, ${ }^{35}$ this study finding a median difference of 678 steps/day. Fourth, our data show improvements in blood pressure and endothelial function in the treatment arm, suggesting BRJ impacts on the risk of vascular events in COPD which are known to be elevated. ${ }^{36}$ We performed an additional responder analysis. Taking a threshold of $35 \mathrm{~m}$ the number of responders in the active arm was $39(68.4 \%)$ and in the placebo group 29 $(44.6 \%)\left(\chi^{2}=6.98, \mathrm{p}=0.008\right)$.

Other supplements have been studied in COPD. A daily dose of vitamin D (1200 IU vitamin $\mathrm{D}_{3} /$ day) for 6 months was found to confer no improvement in 6 min walk test. ${ }^{37}$ A carbohydraterich supplement containing $560 \mathrm{kcal}$ of carbohydrates taken daily for the duration of a 7-week programme of PR had no impact on either peak exercise capacity (ISWT) or exercise endurance (measured by the endurance shuttle walk test). ${ }^{38} \mathrm{~A}$ systematic review and meta-analysis found that creatine supplementation in COPD subjects does not improve exercise capacity in individuals undergoing PR. ${ }^{39}$ ACE inhibition reduced blood pressure, but actually attenuated the improvement in peak work rate after completion of PR. ${ }^{40}$

Cardiovascular comorbidity is common in $\mathrm{COPD}^{36}$ and vascular responsiveness measured using FMD of the brachial artery is known to be significantly impaired in individuals with COPD compared with controls. ${ }^{41}$ It has been shown that dietary nitrate supplementation can reduce blood pressure in healthy individuals, ${ }^{42}$ older adults, ${ }^{14}$ peripheral arterial disease ${ }^{43}$ and COPD, ${ }^{6-8}$ though studies to date have been of short duration. Our finding that this effect on blood pressure is sustained over at least 8 weeks suggests that this intervention should be investigated further for possible impacts on cardiovascular events in COPD and other long-term conditions, over and above impacts on exercise capacity and physical performance. The improvement in endothelial function we observed is consistent with another study showing that dietary nitrate supplementation improved it in people with hypercholesterolaemia, with an increase in FMD $(1.1 \%(0.26), \mathrm{p}=0.003){ }^{44}$

There are multiple potential NO-related mechanisms that may have contributed an increase in exercise capacity following dietary nitrate supplementation, which are reviewed in detail elsewhere. ${ }^{45}$ These include increased skeletal muscle oxygen delivery through enhanced blood flow to muscle, ${ }^{5}$ reduced ATP cost to generate a submaximal force, increased mitochondrial efficiency by reducing the oxygen cost of mitochondrial ATP resynthesis ${ }^{45}$ and improved calcium handling in fasttwitch muscle fibres resulting in increased contractile force. We have shown previously that nitrate supplementation reduces 
the oxygen cost of exercise in people with $\mathrm{COPD},{ }^{6}$ a condition known to be associated with skeletal muscle abnormalities including fibre-type switch and mitochondrial dysfunction. ${ }^{47-51}$ In addition, effects on systemic and pulmonary circulation may improve cardiovascular function. In the present trial, nitrate supplementation may have enhanced the training effect of PR by increasing the level at which individuals were able to exercise, or by augmenting the training effect at an equivalent level of exertion. Alternatively, one or more effects of repeated dosing may have persisted beyond the end of the PR and dosing period. Importantly, since the outcome measures were made 7-14 days following the last dose of BRJ, our findings cannot be due to an acute effect of dietary nitrate supplementation as the half life of nitrate in the body is less than 24 hours. ${ }^{15}$

\section{Methodological issues}

The study made use of standard measures used in PR programmes already running and three of the four sites were district general hospitals (DGH). By design, endothelial function outcomes were only measured at one site. An advantage of the use of standard PR programmes at DGH sites increases our confidence that the effects of nitrate supplementation are likely to be generalisable.

The completion rate for PR was $74 \%$, similar to the $68 \%$ seen in the 2018 Royal College of Physicians PR audit, ${ }^{52}$ and the dropout rates did not differ significantly between groups, suggesting that dietary nitrate supplementation is safe.

A strength of the study is the closely matched placebo, as the nitrate-deplete BRJ matched the nitrate-rich BRJ in every way except the nitrate content. The supplements were consumed without supervision. To check compliance empty bottles were collected and recorded prior to each PR session. Participants were also advised to avoid nitrate-rich foodstuffs 48 hours prior to the study visits at the beginning and end of the trial and avoid antibacterial mouthwash and chewing gum. It is unlikely that this differed between groups in a way that might have influenced the results. The fall in blood pressure observed in the treatment group supports the notion that participants were adherent to treatment. We abandoned initial plans to measure blood nitrate levels because of logistical difficulties which could have provided further evidence about compliance. Measurement of salivary nitrate and nitrite content could also have been an option.

\section{CONCLUSION}

Dietary nitrate supplementation in combination with PR increased exercise capacity, lowered blood pressure and improved endothelial function in people with COPD compared with placebo. Further longer term trials are needed to see if these benefits are sustained over time.

Twitter Matthew J Pavitt @DrMattPav, Adam Lewis @apl104,William Man @ toplungdoc and Nicholas S Hopkinson @COPDdoc

Acknowledgements The authors thank the Pulmonary Rehabilitation staff for their assistance in undertaking this study. The authors also thank all the trial participants and also express their thanks to the Moulton Charitable Foundation for funding this study.

Contributors NSH and MIP developed the original idea for the research study. NSH, MJP and RJT wrote the study protocol. WASB designed the statistical analysis plan. MJP, KJC, RJT, SB, AL, BM and HJ undertook patient visits and collected trial data. WM, SH, KS and DS were local site primary investigators. MJP analysed the data and wrote the first draft of the manuscript. All authors edited and contributed to the final manuscript.

Funding Moulton Charitable Foundation Grant.

Disclaimer The Moulton Charitable Foundation played no role in the conduct or analysis of this study.
Competing interests None declared.

\section{Patient consent for publication Not required.}

Ethics approval All participants provided written informed consent prior to enrolment in the study, which was approved by the London Bridge Health Research and Ethics Committee (Ref: 14/LO/1474) and conducted in line with the principles of the Declaration of Helsinki.

Provenance and peer review Not commissioned; externally peer reviewed.

Data availability statement Data are available upon reasonable request. Individual participant data that underlie the results in the article, after deidentification (text, tables, figures and appendices), will be available. The study protocol and statistical analysis plan will be available. Data will be made immediately available following publication; no end date. Data will be available to anyone who wishes to access the data, for any purpose. Data will be available indefinitely.

\section{ORCID iDs}

Matthew J Pavitt http://orcid.org/0000-0001-9390-3070

Nicholas S Hopkinson http://orcid.org/0000-0003-3235-0454

\section{REFERENCES}

1 Rochester CL, Vogiatzis I, Holland AE, et al. An official American thoracic Society/ European respiratory Society policy statement: enhancing implementation, use, and delivery of pulmonary rehabilitation. Am J Respir Crit Care Med 2015;192:1373-86.

2 Philip K, Gaduzo S, Rogers J, et al. Patient experience of COPD care: outcomes from the British lung Foundation patient Passport. BMJ Open Respir Res 2019;6:e000478.

3 Stamler JS, Meissner G. Physiology of nitric oxide in skeletal muscle. Physiol Rev 2001;81:209-37.

4 Larsen FJ, Weitzberg $E$, Lundberg JO, et al. Effects of dietary nitrate on oxygen cost during exercise. Acta Physiol 2007;191:59-66.

5 Bailey SJ, Winyard P, Vanhatalo A, et al. Dietary nitrate supplementation reduces the 02 cost of low-intensity exercise and enhances tolerance to high-intensity exercise in humans. J Appl Physiol 2009;107:1144-55.

6 Curtis KJ, O'Brien KA, Tanner RJ, et al. Acute dietary nitrate supplementation and exercise performance in COPD: a double-blind, placebo-controlled, randomised controlled pilot study. PLoS One 2015;10:e0144504.

7 Berry MJ, Justus NW, Hauser J, et al. Dietary nitrate supplementation improves exercise performance and decreases blood pressure in COPD patients. Nitric Oxide 2015;48:22-30.

8 Kerley CP, Cahill K, Bolger K, et al. Dietary nitrate supplementation in COPD: an acute, double-blind, randomized, placebo-controlled, crossover trial. Nitric Oxide 2015:44:105-11.

9 Kerley CP, James PE, McGowan A, et al. Dietary nitrate improved exercise capacity in COPD but not blood pressure or pulmonary function: a 2 week, double-blind randomised, placebo-controlled crossover trial. Int J Food Sci Nutr 2019;70:222-31.

10 Leong P, Basham JE, Yong T, et al. A double blind randomized placebo control crossover trial on the effect of dietary nitrate supplementation on exercise tolerance in stable moderate chronic obstructive pulmonary disease. BMC Pulm Med 2015;15:52.

11 Beijers RJHCG, Huysmans SMD, van de Bool C, et al. The effect of acute and 7-days dietary nitrate on mechanical efficiency, exercise performance and cardiac biomarkers in patients with chronic obstructive pulmonary disease. Clin Nutr 2018;37:1852-61.

12 Shepherd Al, Wilkerson DP, Dobson L, et al. The effect of dietary nitrate supplementation on the oxygen cost of cycling, walking performance and resting blood pressure in individuals with chronic obstructive pulmonary disease: a double blind placebo controlled, randomised control trial. Nitric Oxide 2015;48:31-7.

13 Vogelmeier CF, Criner GJ, Martinez FJ, et al. Global strategy for the diagnosis, management, and prevention of chronic obstructive lung disease 2017 report. gold executive summary. Am J Respir Crit Care Med 2017:195:557-82.

14 Kelly J, Fulford J, Vanhatalo A, et al. Effects of short-term dietary nitrate supplementation on blood pressure, 02 uptake kinetics, and muscle and cognitive function in older adults. Am J Physiol Regul Integr Comp Physiol 2013;304:R73-83.

15 Webb AJ, Patel N, Loukogeorgakis S, et al. Acute blood pressure lowering, vasoprotective, and antiplatelet properties of dietary nitrate via bioconversion to nitrite. Hypertension 2008;51:784-90.

16 Woessner M, Smoliga JM, Tarzia B, et al. A stepwise reduction in plasma and salivary nitrite with increasing strengths of mouthwash following a dietary nitrate load. Nitric Oxide 2016:54:1-7.

17 Warren GL, Park ND, Maresca RD, et al. Effect of caffeine ingestion on muscular strength and endurance: a meta-analysis. Med Sci Sports Exerc 2010;42:1375-87.

18 Spruit MA, Singh SJ, Garvey C, et al. An official American thoracic Society/European respiratory Society statement: key concepts and advances in pulmonary rehabilitation. Am J Respir Crit Care Med 2013;188:e13-64.

19 Dodd JW, Hogg L, Nolan J, et al. The COPD assessment test (cat): response to pulmonary rehabilitation. A multicentre, prospective study. Thorax 2011;66:425-9.

20 Puhan MA, Frey M, Büchi S, et al. The minimal important difference of the hospital anxiety and depression scale in patients with chronic obstructive pulmonary disease. Health Qual Life Outcomes 2008;6:46 
21 Steiner MC, Barton RL, Singh SJ, et al. Bedside methods versus dual energy $\mathrm{X}$-ray absorptiometry for body composition measurement in COPD. Eur Respir J 2002;19:626-31.

22 Rabinovich RA, Louvaris Z, Raste Y, et al. Validity of physical activity monitors during daily life in patients with COPD. Eur Respir J 2013;42:1205-15.

23 Van Remoortel H, Raste Y, Louvaris Z, et al. Validity of six activity monitors in chronic obstructive pulmonary disease: a comparison with indirect calorimetry. PLoS One 2012; 7:e39198.

24 Watz H, Waschki B, Meyer T, et al. Physical activity in patients with COPD. Eur Respir J 2009;33:262-72.

25 Watz H, Waschki B, Boehme C, et al. Extrapulmonary effects of chronic obstructive pulmonary disease on physical activity: a cross-sectional study. Am J Respir Crit Care Med 2008;177:743-51.

26 Manini TM, Everhart JE, Patel KV, et al. Daily activity energy expenditure and mortality among older adults. JAMA 2006;296:171-9.

27 Miller MR, Hankinson J, Brusasco V, et al. Standardisation of spirometry. Eur Respir J 2005:26:319-38.

28 Gibson GJ. Standardised lung function testing. Eur Respir J 1993;6:155-7.

29 Rodriguez-Miguelez P, Seigler N, Harris RA. Ultrasound assessment of endothelial function: a technical guideline of the flow-mediated dilation test. J Vis Exp 2016. doi:10.3791/54011. [Epub ahead of print: 27 Apr 2016].

30 Singh SJ, Jones PW, Evans R, et al. Minimum clinically important improvement for the incremental shuttle walking test. Thorax 2008;63:775-7.

31 Evans RA, Singh SJ. Minimum important difference of the incremental shuttle walk test distance in patients with COPD. Thorax 2019;74:994-5.

32 Zoumot Z, Jordan S, Hopkinson NS. Emphysema: time to say farewell to therapeutic nihilism. Thorax 2014;69:973-5.

33 Steiner MMV, Lowe D, Saleem Khan M, et al. Pulmonary rehabilitation: beyond breathing better. National chronic obstructive pulmonary disease (COPD) audit programme: outcomes from the clinical audit of pulmonary rehabilitation servies in England 2015: national supplementary report. London: RCP, 2017.

34 Mereles D, Ehlken N, Kreuscher S, et al. Exercise and respiratory training improve exercise capacity and quality of life in patients with severe chronic pulmonary hypertension. Circulation 2006;114:1482-9.

35 Demeyer H, Burtin C, Hornikx M, et al. The minimal important difference in physical activity in patients with COPD. PLOS One 2016;11:e0154587.

36 Bloom Cl, Ricciardi F, Smeeth L, et al. Predicting COPD 1-year mortality using prognostic predictors routinely measured in primary care. BMC Med 2019;17:73.

37 Rafiq R, Prins HJ, Boersma WG, et al. Effects of daily vitamin D supplementation on respiratory muscle strength and physical performance in vitamin D-deficient COPD patients: a pilot trial. Int J Chron Obstruct Pulmon Dis 2017;12:2583-92.
38 Steiner MC, Barton RL, Singh SJ, et al. Nutritional enhancement of exercise performance in chronic obstructive pulmonary disease: a randomised controlled trial. Thorax 2003;58:745-51.

39 Al-Ghimlas F, Todd DC. Creatine supplementation for patients with COPD receiving pulmonary rehabilitation: a systematic review and meta-analysis. Respirology

40 Curtis KJ, Meyrick VM, Mehta B, et al. Angiotensin-Converting enzyme inhibition as an adjunct to pulmonary rehabilitation in chronic obstructive pulmonary disease. Am J Respir Crit Care Med 2016;194:1349-57.

41 Vaes AW, Spruit MA, Theunis J, et al. Endothelial function in patients with chronic obstructive pulmonary disease: a systematic review of studies using flow mediated dilatation. Expert Rev Respir Med 2017;1:1-11.

42 Wylie LJ, Kelly J, Bailey SJ, et al. Beetroot juice and exercise: pharmacodynamic and dose-response relationships. J App/ Physiol 2013;115:325-36.

43 Kenjale AA, Ham KL, Stabler T, et al. Dietary nitrate supplementation enhances exercise performance in peripheral arterial disease. J App/ Physiol 2011;110:1582-91.

44 Velmurugan S, Gan JM, Rathod KS, et al. Dietary nitrate improves vascular function in patients with hypercholesterolemia: a randomized, double-blind, placebo-controlled study. Am J Clin Nutr 2016;103:25-38.

45 Jones AM, Thompson C, Wylie LJ, et al. Dietary nitrate and physical performance. Annu Rev Nutr 2018;38:303-28.

46 Larsen FJ, Schiffer TA, Borniquel S, et al. Dietary inorganic nitrate improves mitochondrial efficiency in humans. Cell Metab 2011;13:149-59.

47 Farre-Garros R, Lee JY, Natanek SA, et al. Quadriceps miR-542-3p and -5p are elevated in COPD and reduce function by inhibiting ribosomal and protein synthesis. $J$ Appl Physiol 2019;126:1514-24.

48 Maddocks M, Shrikrishna D, Vitoriano S, et al. Skeletal muscle adiposity is associated with physical activity, exercise capacity and fibre shift in COPD. Eur Respir J 2014;44:1188-98.

49 Patel MS, Lee J, Baz M, et al. Growth differentiation factor-15 is associated with muscle mass in chronic obstructive pulmonary disease and promotes muscle wasting in vivo. J Cachexia Sarcopenia Muscle 2016;7:436-48.

50 Hopkinson NS, Sharshar T, Ross ET, et al. Corticospinal control of respiratory muscles in chronic obstructive pulmonary disease. Respir Physiol Neurobiol 2004;141:1-12.

51 Jackson AS, Shrikrishna D, Kelly JL, et al. Vitamin D and skeletal muscle strength and endurance in COPD. Eur Respir J 2013;41:309-16.

52 Steiner MMV, Lowe D, Holzhauer-Barrie J, et al. Pulmonary rehabilitation: an exercise in improvement. National chronic obstructive pulmonary disease (COPD) audit programme: clinical and organisational audits of pulmonary rehabilitation services in Engladn and Wales 2017. National report. London: RCP, 2018. 